\title{
La transferencia tecnológica para el desarrollo de guías de práctica clínica informadas en evidencia
}

\author{
The transfer knowledge for the development of clinical guidelines informed on \\ evidences
}

\author{
Gloria Carmona Clavijo ${ }^{1,2, a}$, Catherine Bonilla Untiveros ${ }^{1,2, b}$, Nora Reyes Puma ${ }^{1,2, \mathrm{~d}}$, \\ Karen Huamán Sánchez ${ }^{1, \mathrm{c}}$, Celia Castillo Villacrez ${ }^{1,2, \mathrm{e}}$, Patricia Caballero Nopo ${ }^{1,2, f}$ \\ Unidad de Análisis y Generación de Evidencias en Salud Pública (UNAGESP), Instituto Nacional de Salud. Lima, Perú. \\ 2 Universidad Nacional Mayor de San Marcos. Lima, Perú. \\ ${ }^{a}$ Master in Science. Lic. en obstetricia \\ ${ }^{b}$ Master in Science. Lic. en nutrición \\ c Lic. en enfermería \\ ${ }^{d}$ Magister en Salud Pública. Médico \\ e Master en Science. Médico intensivista \\ Master in Science. Médico infectólogo.
}

Correspondencia:

Gloria Carmona Clavijo.

Calle Santa Felicidad 222. Pando 3ra

etapa. Cercado de Lima.

944477951

gloriacarmona.c@gmail.com

Recibido: 14 de septiembre 2018 Aprobado: 14 de noviembre 2018 Conflictos de interés: Los autores declaran no tener conflictos de interés..

Fuentes de financiamiento: Instituto Nacional de Salud.

Citar como: Carmona G, Bonilla C, Reyes N, Huamán K, Castillo C, Caballero P. La transferencia tecnológica para el desarrollo de guías de práctica clínica informadas en evidencia. An Fac med. 2018;79(4):360-6.

DOI: http://dx.doi.org/10.15381/anales. v79i4.15644
An Fac med. 2018; 79(4):360-6 / DOI: http://dx.doi.org/10.15381/anales.v79i4.15644

\section{Resumen}

Este artículo comparte la experiencia del proceso de transferencia tecnológica (TT) realizada por el Instituto Nacional de Salud (INS) hacia los grupos clínicos multidisciplinarios de las diversas IPRESS y direcciones del Ministerio de Salud (MINSA), dentro del marco de desarrollo de guías de práctica clínica informadas en evidencia (GPC-IE). Presenta el contexto evolutivo de las GPC junto al rol que desarrolló el INS, tanto en el desarrollo de la normativa vigente como en su implementación. Expresa las situaciones propias de la operacionalización del proceso que no fueron contempladas inicialmente en la norma y los aportes realizados para su abordaje; como la variabilidad de las estrategias de priorización de temas a desarrollar, las características adicionales del grupo elaborador (GE) y grupo metodólogo (GM), la relevancia del grupo gestor, las estrategias pedagógicas empleadas para el manejo de los grupos clínicos, las precisiones adicionales que se realizaron en relación a la declaración de conflicto de interés $(\mathrm{DCl})$ en pro de contribuir en la transparencia, y de fortalecer la TT asumida. Manifiesta las estrategias empleadas para la valoración de las perspectivas y preferencias de los pacientes. Finalmente expresa los resultados y los desafíos en la TT continua en el marco de GPC-IE.

Palabras clave: Guía de Práctica Clínica; Práctica Clínica Basada en la Evidencia; Perú

\section{Abstract}

This article shares the experience of the technology transfer (TT) developed by the Peruvian National Institute of Health (INS) towards the multidisciplinary clinical groups of different IPRESS and managers of the Ministry of Health (MINSA), inside the framework of the development of guidelines for Clinical Practice-Informed in Evidence (GPC-IE). It presents the evolutionary context of the CPG together with the role assumed by the INS, in their development of current regulations and in their implementation. It expresses the inherent situation to operationalization of the process that were not initially contemplated in the guideline and the contributions made to afford it; as the variability of the prioritization strategies of topics to be developed, the additional characteristics of the development group (GE) and methodology group (GM), the relevance of the management group, the pedagogical strategies used for the management of the clinical groups, the additional precisions that were carried out in relation to the declaration of conflict of interest (DCI) in order to contribute to transparency, and to strengthen the assumed TT. It shows the strategies used to assess the perspectives and preferences of patients. Finally it expresses the results and the challenges in the continuous TT in the framework of GPC-IE.

Keywords: Practice Guideline; Evidence-Based Practice; Peru 


\section{INTRODUCCIÓN}

En nuestro país, las GPC se elaboran para estandarizar y brindar cobertura a la atención de salud de los pacientes que presentan enfermedades de prioridad sanitaria a nivel nacional o institucional. Las mencionadas GPC han evolucionado en la metodología para su desarrollo y los fines de su implementación, incorporando el uso de herramientas de análisis y síntesis de evidencias científicas, por el interés de las instituciones financiadoras o aseguradoras en salud, por la estrategia del Ministerio de Economía para asignar el presupuesto estatal y por el derecho a la salud que es vigilado por la sociedad civil y el estado. Ante la nueva norma técnica que promueve el uso de evidencias científicas y herramientas metodológicas para elaborar guías, existe una brecha de implementación y entre los factores que limitan su utilización se encuentra la carencia de masa crítica con competencias suficientes y tiempo disponible en las instituciones; además de la inestabilidad en la gestión para el desarrollo de guías. Como consecuencia, existen guías nacionales antiguas, desactualizadas, de baja calidad metodológica y de modo paralelo un creciente número de guías institucionales adoptadas con escasa metodología, que se duplican entre instituciones, generando mayor inequidad y gran variabilidad $^{2}$. En ese sentido, diversos manuscritos nacionales ${ }^{2,3}$ e internacionales ${ }^{4,5}$ han planteado y difundido la necesidad del desarrollar GPC-IE. El Instituto Nacional de Salud (INS) a través de la UNAGESP, promueve el uso de la evidencia científica realizando de manera articulada diversos productos y actividades ${ }^{6}$. Así, en el 2013 recibió el encargo del MINSA de desarrollar y proponer la estructura metodológica de la Norma Técnica para la Elaboración de GPC vigente desde setiembre del 2015 hasta la fecha, con lo cual se regula su desarrollo7,8. Posterior el ello, asumió el rol de fortalecer el proceso de implementación de la referida norma técnica a través de la TT, trabajando de manera directa y sistemática con equipos clínicos de diversos establecimientos de salud. Este rol, que resulta enriquecedor, sistemático y técnico, tanto para los clínicos participantes como para los metodólogos que la impartimos, aún dista de la necesidad de contar con suficientes GPC-IE de calidad en nuestro país que colaboren en la toma de decisiones sobre la atención en salud más apropiada en base a la evidencia y optimizando el uso de los recursos. Es por ello que este manuscrito presenta la metodología empleada en el proceso de TT y formación de masa crítica para el desarrollo de GPC-IE, las lecciones aprendidas, los aportes realizados en la práctica adicionales a la normativa vigente, los resultados y los desafíos identificados.

\section{METODOLOGÍA PARA EL DESARROLLO DE GPC}

Para el diseño del programa de TT se consideró y aplicó la metodología expresada en la norma técnica, no obstante, estos pasos metodológicos no necesariamente son bien entendidos por todos los que la estudian, resultando un desafío para su compresión y aplicación. Por ello, la estrategia fue diseñar un programa de transferencia que se ha caracterizado por la simplificación de las herramientas metodológicas para facilitar su comprensión y aprendizaje. Ello significó la creación de instrumentos, la inclusión de pasos adicionales y la incorporación de otras metodologías de uso de evidencias y de consenso, desarrolladas en las siguientes etapas de este manuscrito.

Tabla 1. Resultado de priorización en salud neonatal empleando el instrumento FOR-CNSP-415 para priorización de temas en el campo de la salud

\begin{tabular}{llc} 
Posición obtenida & Temas de salud neonatal priorizados & Puntaje obtenido \\
\hline 1 & Enfermedad de membrana hialina & 2290 \\
\hline 2 & Asfixia neonatal & 2206 \\
\hline 3 & Taquipnea transitoria & 2113 \\
\hline 4 & Hipoglicemia & 2045 \\
\hline 5 & Ictericia neonatal & 2005 \\
\hline 6 & Soporte nutricional & 1985 \\
\hline 7 & Seguimiento recién nacido de alto riesgo & 1984 \\
\hline 8 & Atención integral del RN prematuro & 1960 \\
\hline 10 & Síndrome de aspiración meconial & 1843 \\
\hline 11 & Neumonía congénita & 1838 \\
\hline 12 & Trastorno hidroelectrolítico & 1696 \\
\hline 13 & Toxoplasmosis & 1646 \\
\hline
\end{tabular}

Fuente: Taller de priorización realizado a solicitud de Dirección por Etapas de Vida del componente salud neonatal del MINSA, realizado en enero de 2018 en el Instituto Nacional de Salud del Niño, Sede San Borja, con la participación de pediatras de diversos hospitales del MINSA. 
Lima convocados por la Dirección de Etapa de Vida Niño y Componente Neonatal. Actualmente este formulario se encuentra libre en línea y puede ser aplicado por los diversos grupos elaboradores de GPCIE capacitados.

Sin embargo, este sistema puede variar. Por ejemplo, en el campo de la salud materna la priorización estuvo basada en el consenso según la carga de mortalidad y morbilidad durante la gestación y el puerperio. En otras situaciones, como por ejemplo en las GPC sobre la artritis idiopática juvenil o fibrosis quística, esta selección se justificó en relación a la necesidad de disminuir la variabilidad en la atención y poder incrementar sus coberturas de atención en la detección temprana, diagnóstico y tratamiento. Esto nos manifiesta que a nivel del MINSA no hay un patrón homogéneo en relación a la priorización; además, la priorización no se encuentra a cargo de una sola dependencia u oficina.

\section{CONFORMACIÓN DE LOS GRUPOS ELABORADORES DE LAS GPC}

Con el tema ya definido, se procede con la conformación del GE, el cual es seleccionado por los gestores de la oficina de calidad de las instituciones de salud o por los comités de expertos nacionales del MINSA. Para ello, además de los requisitos considerados en la normativa vigente, se ha encontrado importante para la adherencia al proceso de TT: la capacidad de lectura crítica en inglés, considerando que el grosor de la evidencia científica es publicado en ese idioma; estar conformado por clínicos multidisciplinares "seniors" y "middles" con experiencia en el tema propuesto, que laboren en establecimientos de salud e institutos y/o profesionales que pertenecen oficialmente a los comités nacionales de expertos del MINSA; tener apertura al uso de tecnologías virtuales como gestor de referencias como nubes de almacenamiento virtual; y contar con representantes de los diversos niveles de atención e instituciones. Por sobre todo, el tener voluntad y apertura a la discusión de que en algunas situaciones lo que sustenta la evidencia puede no estar alineada con la práctica médica actual, representando una oportunidad para fortalecer la atención de salud basada en evidencia actual. Este conjunto de características permite una transferencia de competencias cruzadas dirigida por el GM y fortalece la discusión desde diversas perspectivas permitiendo una retroalimentación constante.

En relación a los gestores, tanto del INS como de cada una de las instituciones de salud participantes, evidenciamos que por su experiencia conocen el sistema de salud tanto en el campo asistencial y administrativo así como en el proceso de gestión. Asimismo, tienen apertura a incorporar las herramientas metodológicas que fortalezca el rigor y la calidad de los productos (en este caso GPC) de la gestión en la que se encuentran, a fin de que estos productos sean sustentables y sostenibles para que de ese modo les permita gestionar los recursos que consideren necesarios en beneficio de la salud de los peruanos.

El GM si bien tiene un coordinador - líder en cada GPC, es un equipo que también ha presentado características particulares en este proceso de "aprender haciendo" ${ }^{10}$, para lo cual además de conocer y cumplir lo mencionado en la norma técnica y tener experiencia en investigación, se han desarrollado y obtenido competencias metodológicas a través de estrategias de docencia ${ }^{11}$, de manejo asertivo de grupos a fin de obtener el mejor aporte de cada uno de los miembros elaboradores participantes. Este proceso de capacitación continua, ha sido monitorizado y refinado gracias al soporte que nos han brindado nuestros pares metodólogos de la Organización Panamericana de la Salud y del Grupo desarrolladores de GPC de la Universidad de MC Master (Canadá).

Es así que en estos procesos paralelos se asume la TT, lideradas en una primera oportunidad por la UNAGESP. Sin embargo, en una segunda oportunidad, cuando el GE, ya cuenta con expertos clínicos y metodológicos asumiendo un rol de dirección y organización sobre la GPC a desarrollar, la UNAGESP asume un rol de asesoría en lugar de liderazgo técnico. Como ejemplo tenemos a miembros GE de la GPC sobre preclampsia y eclampsia, quienes luego de concluir la referida guía ${ }^{12}$, asumieron el rol de liderazgo con perfiles mixtos (tanto clínico como metodológico) en la GPC-IE sobre sepsis obstétrica.

Una vez conformado el GE, organizado el funcionamiento del grupo y obtenida la DCl desde el inicio del proceso de trabajo, se decide el alcance de la guía según lo describe la norma. En relación a la $\mathrm{DCl}$ se consideró por conveniente, según lo observado en los perfiles de los GE, realizar modificaciones al formato a fin de obtener una declaración más completa y pertinente a través del Sistema de Gestión de la Calidad del INS en el cual se pregunta adicionalmente sobre otros aspectos como el financiamiento en formación académica y capacitaciones. Estas declaraciones también son recolectadas en la formulación de las recomendaciones y sus contenidos son analizados por el equipo metodológico a fin de definir la participación más oportuna y pertinente de cada uno de los miembros del panel de expertos. Este proceso, el cual es muy singular y particular en cada caso, no busca excluir participaciones, busca orientar el mejor aporte que podrá proporcionar cada participante. Por ejemplo, se podría decidir que el participante tenga voz por su experiencia clínica y sin voto según la pregunta clínica que se esté abordando.

Obtenido estos aspectos, se continua con la selección de GPC que permita tomar la decisión de preparar una guía de novo o adaptar, según NT. En aras de optimizar la dinámica del proceso y del tiempo de elaboración, las GPC identificadas son clasificadas según la metodología utilizada con un instructivo elaborado para tal fin; así, las GPC pueden ser de tres tipos: por consenso formal, informal o informado en evidencia a través del instrumento desarrollado y validado por el Centro Nacional de Salud Pública ${ }^{13}$. Posteriormente las GPC informadas en evidencia son verificadas a través una lista de chequeo para identificar el cumplimiento de los criterios para ser evaluadas por pares con la herramienta AGREE $\|^{14}$, seleccionando finalmente la de mayor calidad metodológica y que responda mejor a las necesidades. El GE es entrenado por el GM en el uso de estas herramientas para realizar el proceso de manera conjunta. 
FORMULACIÓN Y AJUSTE DE LAS PREGUNTAS PICO (POBLACIÓN, INTERVENCIÓN, COMPARACIÓN, OUTCOME)

Este proceso se realiza de dos maneras: 1 . Se analiza la pertinencia y potencial inclusión de alguna o varias de las preguntas consideradas en la GPC seleccionada. Seguido a ello, los clínicos plantean preguntas de novo que consideran pertinente de atención en nuestro contexto local y que son ajenas a la realidad de la GPC seleccionada. 2. Se realiza la formulación de las preguntas y luego se compara la similitud con aquellas presentadas en la GPC seleccionada. En ambos casos, durante esta fase, los clínicos, por su experiencia y su entrenamiento heterogéneo en relación a la metodología para el desarrollo de GPC, suelen proponer diversas preguntas que al ser traducidas al formato PICO pueden corresponder a: necesidades de investigación primaria en su campo de acción; al uso de herramientas de diagnóstico, terapias o fármacos no disponibles en nuestro medio; procedimientos de atención en salud que requieren ser sustentados con evidencia científica o a la práctica operativa de los flujogramas de acción. En ese sentido, se requiere de un proceso de análisis y priorización de PICOs, utilizando una lista de ajuste y relevancia (tabla 2) a través de la discusión y reflexión con el GE. Este ejercicio permite disminuir la posibilidad de sesgo hacia un tema específico y fomenta la discusión en relación a la pertinencia de la pregunta considerando lo disponible localmente, resaltando la relevancia de los flujogramas de atención por permitir evidenciar la secuencia de la atención clínica desde la semiología en el tema de salud en estudio. Los flujogramas oficiales de atención, en caso los hubiera, son actualizados por el GE para este proceso; en el caso de no contar con flujogramas, estos son elaborados en conjunto.

\section{ACTUALIZACIÓN DE LAS ESTRATEGIAS DE BÚSQUEDA Y SELECCIÓN POR TÍTULO Y RESUMEN}

Es en esta fase donde se realiza el entrenamiento en búsqueda sistemática de evidencia científica publicada en bases de datos como MEDLINE (PubMed), Embase (Ovid), The Cochrane Library y LILACS. Además, esta acción promueve el uso de la evidencia sistematizada, el cual es una de las funciones que asume la UNAGESP

Tabla 2. Criterios para ajuste de preguntas PICO (población, intervención, comparación, outcome)

\begin{tabular}{|c|c|c|c|c|}
\hline \multirow[b]{3}{*}{ Pregunta clínica } & \multicolumn{3}{|c|}{ Preguntas de la GPC } & \multirow[b]{3}{*}{$\begin{array}{l}\text { Resultado o } \\
\text { desenlace }\end{array}$} \\
\hline & \multicolumn{3}{|c|}{ Pregunta en formato PICO } & \\
\hline & Población & Intervención & Comparación & \\
\hline $\begin{array}{l}\text { Relevancia clínica } \\
\text { de la pregunta }\end{array}$ & muy relevante & Relevante & $\begin{array}{l}\text { poco } \\
\text { relevante }\end{array}$ & no relevante \\
\hline \multicolumn{5}{|c|}{ Ajuste de pregunta PICO } \\
\hline $\begin{array}{l}\text { ¿Se ubica en el } \\
\text { flujograma de } \\
\text { atención? }\end{array}$ & \multicolumn{2}{|c|}{ Si } & \multicolumn{2}{|c|}{ No } \\
\hline $\begin{array}{l}\text { ¿Cómo es la } \\
\text { práctica actual? }\end{array}$ & muy homogénea & homogénea & $\begin{array}{l}\text { poco } \\
\text { heterogénea }\end{array}$ & $\begin{array}{l}\text { muy } \\
\text { heterogénea }\end{array}$ \\
\hline $\begin{array}{l}\text { ¿Que se desea } \\
\text { mejorar? }\end{array}$ & \multirow{4}{*}{ Respuesta abierta } & & & \\
\hline $\begin{array}{l}\text { ¿Qué estamos } \\
\text { innovando? }\end{array}$ & & & & \\
\hline $\begin{array}{l}\text { ¿Cómo se } \\
\text { beneficia al } \\
\text { paciente? }\end{array}$ & & & & \\
\hline $\begin{array}{l}\text { ¿Será factible su } \\
\text { implementación? }\end{array}$ & & & & \\
\hline
\end{tabular}

como centro colaborador Cochrane. En relación a la lectura completa del artículo para determinar su inclusión o exclusión, este ejercicio se realiza primero a manera de ejemplo con todo el GE y luego las preguntas son distribuidas por pares a fin de disminuir los potenciales sesgos y designar responsabilidades específicas en la selección de evidencia y en la obtención de los artículos completos. Los GE se han mostrado muy interesados en el uso de estas herramientas por la utilidad que les representa tanto para su rol clínico como académico, lo que a su vez permite distribuir las responsabilidades de manera específica a cada integrante, siempre con el acompañamiento del GM.

\section{EVALUACIÓN DEL CUERPO DE LA EVIDENCIA SEGÚN GRADE: RELEVANCIA CLÍNICA Y CALIDAD}

Esta evaluación está centrada en el análisis del cuerpo de la evidencia. Cada miembro del GE es designado a realizar la lectura crítica y analítica de los artículos que responden a la PICO y luego en mesa redonda cada miembro presenta el cuerpo la evidencia resaltando el aporte a la pregunta PICO; por su parte el metodólogo explica la valoración de la calidad de la evidencia según GRADE ${ }^{15}$, permitiendo la discusión, reflexión y ratificación por el GE. Este análisis, evaluación de la calidad y aportes relacionados son transcritos en las tablas GRADE para discusión y formulación de las recomendaciones con el panel de expertos, lo cual se realiza a través de un dialogo deliberativo (DD) ${ }^{16}$.

\section{CONSIDERACIONES DE LA POTENCIAL ACEPTABILIDAD Y PREFERENCIAS DE LA POBLACIÓN DIANA DE LA GPC-IE}

Para considerar estos aspectos se realiza una búsqueda local, nacional y regional de evidencias que reflejen la postura de los pacientes y usuarios. Además, para lograr una aproximación más local al contexto de desarrollo de la GPC, se emplean diversas estrategias según su tópico y alcance. Por ejemplo, en el caso de la guía sobre ansiedad en el paciente con cáncer, se invitó a los representantes de la asociación de pacientes del INEN, 
que voluntariamente desearon participar en una parte del DD, para que nos brindaran sus opiniones, perspectivas sobre la atención al paciente y la facilidad de comprensión en relación a la pruebas psicológicas de tamizaje a través de una conversación informal y formal. Esto permitió al GE considerar estas perspectivas, registrando anotaciones de campo, asegurando el anonimato de los pacientes. En el caso de la guía sobre esquizofrenia, la estrategia fue diferente, para ello los psiquiatras durante la consulta clínica, a través de una guía de conversación informal semi-estructurada elaborada de manera conjunta con el GE, consultaron sobre las preferencias de los pacientes que se encontraban en fase controlada o de mantenimiento y de sus cuidadores. Estas consultas fueron en relación a los contenidos de la GPC, como por ejemplo la posibilidad de recibir una terapia farmacológica y psicosocial de manera combinada. Estos aportes de los pacientes son reportados en cuadernos de campo por los clínicos, previo entrenamiento y son sintetizadas en conjunto para poder mostrar las principales perspectivas, que serán consideradas para la formulación de las recomendaciones junto al panel de expertos. Este proceso permite triangular la información.

\section{FORMULACIÓN DE RECOMENDACIONES INFORMADAS EN EVIDENCIA}

En este proceso se suma el panel de expertos multidisciplinario de diversos establecimientos de salud públicos y privados en una o dos sesiones de jornada completa, a través de un DD, en la cual se presenta el cuerpo y análisis de la calidad de la evidencia. Este análisis sumado a la relevante discusión del reporte de la experiencia clínica y de la situación actual del sistema de salud relacionado a la patología o condición clínica considerada en la GPC por parte del panel de expertos, permite definir la contextualización en la adaptación o exclusión de las recomendaciones de novo, incluyendo la fuerza de la recomendación y el nivel de evidencia que la sostiene ${ }^{11,17}$.

\section{REDACCIÓN, VALIDACIÓN Y APROBACIÓN DE LA GPC-IE}

Una vez obtenidas las recomendaciones por el GE y el panel de expertos, se realiza la redacción extensa de la GPC-IE considerando las especificidades obtenidas en el dialogo, que están enmarcadas principalmente en las consideraciones para la implementación de las recomendaciones sostenidas por evidencia o por el consenso clínico. Seguido a ello se comparte la versión final a expertos clínicos y metodológicos internacionales y nacionales, cuyas sugerencias, observaciones y aportes son discutidos con el GE para su consideración y justificación. En la experiencia de TT realizada por la UNAGESP-INS, la OPS y la Universidad de Mc Master en Canadá colaboran con la revisión metodológica. En relación a la revisión por expertos clínicos, el GE permanentemente identifica a expertos nacionales y reconocidos entre sus pares en relación a la temática de la GPC-IE desarrollada. Estos expertos a su vez han colaborado de manera técnica y puntual en sus revisiones críticas, las cuales fortalecen y contextualizan mejor la GPC.

La redacción de la versión corta es más puntual y se encuentra estructurada según los las normas dispuestas por el MINSA $^{8}$, la cual es una de las instancias de las que depende su aprobación según el alcance de la GPC. En esta versión se resaltan sobre todo los flujogramas de acción según los niveles de atención.

\section{COMPROMISO DE LAS INSTITUCIONES}

Previo al inicio de cada guía, y como condición para aceptar brindar la TT, el INS sostuvo reuniones con los gestores institucionales para explicarles los detalles del programa de transferencia, el compromiso de las instituciones y sus responsabilidades. Sobre el financiamiento, el INS financia las horas de entrenamiento que brindan sus profesionales y les otorga certificación a los clínicos participantes. Las instituciones financian los materiales, equipos de cómputo utilizados, aulas, servicio de internet, refrigerios. Por otro lado, el compromiso de asistencia continua a lo largo de los talleres presenciales, y de la fase no presencial a través de actividades asignadas y seguimiento virtual, está ligado a las facilidades que se les brinden a los clínicos según su institución de procedencia. Para ello el grupo gestor se encarga de gestionar con anticipación la participación de los miembros del GE. Además, en este proceso se ha considerado la incorporación de incentivos para los profesionales de los GE, los cuales no son monetarios y están basados en el desarrollo profesional, el prestigio de conformar un GE de GPC-IE lo que genera reconocimiento de sus pares en sus propias instituciones y la certificación o diploma como elaborador de que le otorga el INS.

\section{RESULTADOS ESPERADOS}

La producción de GPC informadas en evidencia de mejor calidad, en lugar de cantidad, es uno de los resultados esperados de esta intervención de TT. Sin embargo, no es más ni menos importante que el desarrollo de masa crítica de clínicos y de gestores de los programas de salud pública que usen la evidencia para sus decisiones clínicas y gestoras. Así, a la fecha se han conformado 13 grupos elaboradores en las diversas instituciones de salud (tabla 3) así como la formación de 79 profesionales con perfiles mixtos clínicos y metodológicos, los cuales en una segunda oportunidad podrán asumir también el rol metodológico de una siguiente GPC-IE. También, los 153 profesionales multidisciplinarios que han desarrollado las competencias para analizar y sintetizar las evidencias, que han participado en los paneles para formación de preguntas PICO y en los DD para la formulación de las recomendaciones.

Se observó la interacción proactiva y colaborativa entre los diversos grupos elaboradores, ya sean de la misma especialidad y procedentes de diversos institutos u hospitales, como por ejemplo, en la primera reunión de grupos elaboradores de GPC-IE celebrado en el INS. Por otro lado, la estrategia de trabajar con grupos elaboradores optimiza la sostenibilidad y la calidad de la GPC y promueve la adherencia en la implementación. 
Tabla 3. Grupos elaboradores de GPC formados como producto de la transferencia tecnológica

Institución Grupos elaboradores (GE) /Tópico de la GPC Miembros de GE Panel de expertos

Instituto Nacional Materno Perinata

GE / Preclampsia y eclampsia

\begin{tabular}{|c|c|c|c|}
\hline \multirow{3}{*}{ Instituto Nacional Materno Perinatal } & & & \\
\hline & GE/ Sepsis obstétrica & & 18 \\
\hline & GE/ Sepsis neonatal & 9 & 16 \\
\hline \multirow{2}{*}{ Hospital Víctor Larco Herrera } & GE/ Depresión en adultos & 3 & 12 \\
\hline & GE/ Esquizofrenia en adultos & 4 & 10 \\
\hline \multirow{2}{*}{ Comité de Expertos de Salud Neonatal- MINSA } & GE/ Membrana hialina & 7 & 13 \\
\hline & GE/ Asfixia neonatal & 12 & 12 \\
\hline \multirow{2}{*}{$\begin{array}{l}\text { Hospital Nacional Docente Madre Niño San } \\
\text { Bartolomé }\end{array}$} & GE/ Fibrosis quística & \multirow{2}{*}{10} & 10 \\
\hline & GE/ Hiperplasia adrenal congénita & & en proceso \\
\hline \multirow[t]{2}{*}{ Instituto Nacional de Enfermedades Neoplásicas } & $\begin{array}{l}\text { GE/ Manejo del dolor en pacientes adultos con } \\
\text { cáncer }\end{array}$ & 6 & en proceso \\
\hline & GE/ Ansiedad en pacientes adultos con cáncer & 5 & 15 \\
\hline Hospital de Rehabilitación del Callao & GE/ Lumbago en adulto & 8 & en proceso \\
\hline $\begin{array}{l}\text { Comité de Expertos: Red Peruana de Tuberculosis } \\
\text { Pediátrica }\end{array}$ & GE/ Tuberculosis pediátrica & 6 & 22 \\
\hline Total & 13 GPC-IE & 79 & 153 \\
\hline
\end{tabular}

Fuente: Elaboración propia UNAGESP

\section{DESAFÍOS EN LA TRANSFERENCIA TECNOLÓGICA CONTINUA}

Los GE reconocen y están comprometidos en la importancia del uso de la evidencia y su contextualización para sustentar la práctica clínica actual, que contribuirá en el desempeño de los clínicos asistenciales multidisciplinarios.

Hemos identificado desafíos metodológicos donde se hace necesario traducir o crear un lenguaje homogéneo en relación a las recomendaciones. Por ejemplo, para el clínico que es parte del GE, una recomendación fuerte con evidencia de muy baja calidad son comprensibles, porque ha participado en todo el proceso de TT. Sin embargo, no podemos pretender que todos los profesionales asistenciales asignen la misma interpretación de manera inmediata. Es aquí donde el lenguaje de las evidencias debe concretarse y difundirse al universo de profesionales que la utilizarán en su práctica clínica. En relación a la recolección de las preferencias y perspectivas de los pacientes, en aras de evitar largos tiempos en la recolección, actualmente empleamos conversaciones informales con registros de cuadernos de campo o memoriales analíticos. Sin embargo, sería relevante protocolizar estos procesos a través de estudios cualitativos donde luego de obtener el consentimiento informado se pueda, grabar, transcribir analizar y publicar la información.

Existe necesidad de GPC-IE en las regiones y provincias de nuestro país, por lo cual es necesario incluirlos en este proceso y buscar mejores alternativas tecnológicas y de accesibilidad para agilizar el tiempo y las herramientas del proceso de sistematización, sin descuidar el rigor metodológico que sostiene a la GPC-IE.

La implementación de las recomendaciones va más allá del largo proceso de revisión y resolución de aprobación. Es así que, en la implementación de GPC-IE se requiere de una batería de estrategias que permitan su difusión, discusión, adherencia, a fin de evitar que sea un documento técnico que quede encarpetado en las oficinas o repositorios de las instituciones de salud. Esto incluye identificar junto a los clínicos y gestores cuáles son las limitaciones y fortalezas para elaborar estrategias que permitan de manera progresiva su implementación. Además, requiere identificar los indicadores del proceso y resultado que finalmente reflejen la medida en la que se obtienen los objetivos planteados por la GPC-IE
Los GE, representados por los clínicos de diversos establecimientos de salud que ya han recibido la TT, deberán asumir en las siguientes GPC-IE el liderazgo metodológico y continuar con la formación de masa crítica entre sus pares y sociedades científicas. Anidado a ello, el desafío se extiende en que GE no dependan necesariamente de otra institución pública o privada para el desarrollo de GPC-IE.

Finalmente, todo proceso siempre tiene espacio de mejora y refinamiento por lo cual confiamos que el desarrollo continuo de esta masa crítica de GE de GPC-IE; y que las demás instituciones que colaboramos en el desarrollo de GPC-IE podremos, a través de la experiencia sistematizada refinada y continua, generar mejoras relevantes e innovadoras al proceso actual, en beneficio de la salud de todos los peruanos.

\section{AGRADECIMIENTOS}

Agradecemos la permanente disponibilidad y asesoría metodológica de la Organización Panamericana de la salud a través del Dr. Ludovic Reveiz y su equipo. Agradecemos a los directivos, al equipo asistencial y multidisciplinario por su apo- 
yo, deliberación, reflexión y adherencia al proceso de TT realizada por el INS, de las siguientes IPRESS: Instituto Nacional Materno Perinatal, Instituto Nacional de Enfermedades Neoplásicas, Instituto Nacional de Salud del Niño, Hospital Víctor Larco Herrera, Hospital Nacional Docente Madre Niño San Bartolomé, Hospital de Rehabilitación del Callao. En el MINSA, agradecemos a la Dirección de Prevención y Control de Tuberculosis, a la Red Peruana de Tuberculosis Pediátrica, Atención Integral Neonatal de la Dirección General de Intervenciones Estratégicas de Salud Pública del MINSA por los grupos de clínicos participantes en las GPC sobre membrana hialina y asfixia perinatal.

\section{REFERENCIAS BIBLIOGRÁFICAS}

1. Organización Panamericana de la Salud [Internet] Minsa y OPS/OMS fortalecen capacidades técnicas de profesionales de la salud para elaboración de Guias de Práctica Clínica. [Fecha de acceso 28 de agosto 2018]. Disponible en: https://tinyurl. com/y9xkmfgf

2. Canelo-Aybar C, Balbin G, Perez-Gomez A, Florez ID. Guias de práctica clínica en el Perú: evaluación de su calidad usando el instrumento AGREE II. Rev Peru Med Exp Salud Publica. 2016;33(4):732-8. DOI: 10.17843/rpmesp.2016.334.2559

3. García-Mostajo JA, Alva-Díaz C, Suárez V, Timaná $\mathrm{R}$, Canelo-Aybar C. Desarrollo de guias de práctica clínica basadas en evidencia en Perú desde el sector público. Acta Med Peru. 2017;34(3):203-7

4. National Institute for Health and Care Excellence (NICE) [Internet]. Developing NICE guidelines: the manual. [Fecha de acceso 4 de mayo 2018]. Disponible en: https://tinyurl.com/ossh2ge

5. World Health Organization 2012 WHO [Internet]. WHO handbook for guideline development. RM 302-201(ISBN 978924154844 1). [Fecha de acceso 4 de mayo 2018]. Disponible en: https:// tinyurl.com/yd9c7yl2

6. Gutierrez EL, Piazza M, Gutierrez-Aguado A, Carmona G, Caballero P, Reyes N, et al. Uso de la evidencia en políticas y programas de salud aportes del Instituto Nacional de Salud. Rev Peru Med Exp Salud Publica. 2016;33(3)580-4. DOI:10.17843/ rpmesp.2016.333.2308

7. Ministerio de Salud del Perú [Internet]. Resolución Ministerial N519-2006/MINSA, que aprueba el Documento Técnico "Sistema de Gestión de la Calidad en Salud". [Fecha de acceso 4 de mayo 2018]. Disponible en: https://tinyurl.com/y92tvzy7

8. Ministerio de Salud del Perú [Internet]. Norma técnica de salud para la elaboración de Guías de Práctica Clínica del MInisterio de Salud. [Fecha de acceso 4 de mayo 2018]. Disponible en: https:// tinyurl.com/ybakq9z3

9. Centro Nacional de Salud Pública del Instituto Nacional de Salud. Unidad de Analisis y Generación de Evidencias en Salud Pública. Formulario para la Priorización en la selección de temas de salud para las guias de práctica clinica basadas en evidencia. Centro Nac (ITT-CNSP-415).

10. Foti F, Menghini D, Orlandi E, Rufini C, Crinò A, Spera S, Vicari S, Petrosini L, Mandolesi L. Learning by observation and learning by doing in Prader-Willi syndrome. J Neurodev Disord. 2015;7(1):6. DOI: 10.1186/s11689-015-9102-0
11. Scott SM, Schifferdecker KE, Anthony D, Chao J, Chessman AW, Margo K, Seagrave M, Leong SL. Contemporary Teaching Strategies of Exemplary Community Preceptors - Is Technology Helping? Fam Med. 2014;46(10):776-782. DOI: 25646828

12. Instituto Nacional Materno Perinatal. Ministerio de Salud del Perú [Internet]. Resolucion Directoral 026-2018-DG-INMP/MINSA. [Fecha de acceso 4 de mayo 2018]. Disponible en: https://tinyurl. com/yd4brrye

13. Centro Nacional de Salud Pública del Instituto Nacional de Salud. Unidad de Analisis y Generación de Evidencias en Salud Pública. Formulario para la clasificación de guias de práctica clínica según la metodología aplicada para el análisis de la evidencia. Centro Nac (ITT-CNSP-438)

14. Brouwers MC, Browman G, Cluzeau F, Davis D, Feder G, Graham I, et al. AGREE next steps consortium membership, 2009 [Internet]. [Fecha de acceso 4 de mayo 2018]. Disponible en: https:// tinyurl.com/y8ho4htk

15. Schünemann $H$, Brożek J, Guyatt $G$, Oxman A. GRADE Handbook. Grading of Recommendations, Assessment, Development and Evaluation (GRADE) Working Group. Introduction to GRADE Handbook, 2013 [Internet]. [Fecha de acceso 7 de mayo 2018]. Disponible en: https://tinyurl. com/y99244od

16. Carmona G, Bonilla $\mathrm{C}$, Huamán $\mathrm{K}$, Reyes N, Hijar G, Caballero P. Utilidad de los diálogos deliberativos para la formulación de recomendaciones de guias de práctica clínica. Rev Peru Med Exp Salud Publica. 2017;34(4):738-43. DOI: 10.17843/ rpmesp.2017.344.2886

17. Ministerio de Salud del Perú [Internet]. Resolución Ministerial N526-2011/MINSA, que aprueba las Normas para la Elaboración de Documentos Normativos del Ministerio de Salud. [Fecha de acceso 4 de mayo 2018]. Disponible en: http://bvs.minsa. gob.pe/local/MINSA/3300.pdf 